\title{
Design of Amino Acid- and Carbohydrate-Based Anticancer Drugs to Inhibit PIM kinases, an In Silico Study
}

\author{
Sepideh Kalhor ${ }^{1}$ and Alireza Fattahi ${ }^{1}$ \\ ${ }^{1}$ Sharif University of Technology
}

February 10, 2021

\begin{abstract}
PIM-1 is a serine-threonine kinase which is mainly expressed in tissues such as Thymus, spleen, bone marrow, and liver. This protein takes a role in many stages of the cell cycle, including the regulation of cell cycle progression and apoptosis. According to many studies, overexpression of PIM kinases happens in various types of human tumors; such as lymphomas, prostate cancer, and oral cancer. As a result, the design of drugs to inhibit PIM-1 in cancerous cells has attracted many attentions in recent years. This study aimed to design the alternative inhibitors for PIM-1 kinase, which are based on carbohydrates and amino acids and are expected to be non-toxic and to have the same chemotherapeutic effects as the traditional agents. The combinatorial use of quantum mechanics studies and molecular dynamic simulation (MD) has enabled us to precisely predict the mechanism of the inhibition of PIM-1 kinase by the novel designed drugs and to compare them with the recently synthesized chemotherapeutic drugs; such as DBC.
\end{abstract}

\section{Design of Amino Acid- and Carbohydrate-Based Anticancer Drugs to Inhibit PIM kinases, an In Silico Study}

Sepideh Kalhor, Alireza Fattahi*

Department of Chemistry, Sharif University of Technology, Tehran, Iran

* Corresponding Author: fattahi@sharif.edu

Abstract: PIM-1 is a serine-threonine kinase which is mainly expressed in tissues such as Thymus, spleen, bone marrow, and liver. This protein takes a role in many stages of the cell cycle, including the regulation of cell cycle progression and apoptosis. According to many studies, overexpression of PIM kinases happens in various types of human tumors; such as lymphomas, prostate cancer, and oral cancer. As a result, the design of drugs to inhibit PIM-1 in cancerous cells has attracted many attentions in recent years. This study aimed to design the alternative inhibitors for PIM-1 kinase, which are based on carbohydrates and amino acids and are expected to be non-toxic and to have the same chemotherapeutic effects as the traditional agents. The combinatorial use of quantum mechanics studies and molecular dynamic simulation (MD) has enabled us to precisely predict the mechanism of the inhibition of PIM-1 kinase by the novel designed drugs and to compare them with the recently synthesized chemotherapeutic drugs; such as DBC.Key words: PIM-1, cell cycle progression, apoptosis, cancer cells, amino acid, monosaccharides, molecular dynamic simulation

\section{INTRODUCTION}

PIM-1 is a serine-threonine kinase encoded by a gene with the same name which is located on chromosome 6p21.1-p21.31 in human. This enzyme is classified as a calcium/calmodulin-regulated kinases (CAMK) group $[1,2]$. Thymus, spleen, bone marrow and liver are tissues, in which PIM-1 enzyme is mainly expressed [3]. The PIM-1 protein is found to have existed in both the cytoplasm and the nucleus of cells [4]. According to many studies, PIM-1 kinase involves in many stages of cell life; such as the regulation of cell cycle progression 
and apoptosis [5]. The PIM-1 kinase firstly was found and recognized in the studies related to the Moloney murine leukaemia virus (MoMuLV) in lymphomas in mice [6]. Following that, PIM-2 and PIM-3 were cloned and identified as serine-threonine kinases group, and it has been illustrated that the overexpression of PIM kinases happens in various types of human tumours; such as lymphomas, prostate cancer, and oral cancer [7-11]. Also, it should be mentioned that PIM-1kinase promotes therapeutic resistance in lung cancer [12]. Moreover, it was evident that of the great barrier in the successful treatment of many types of cancers is resistance toward chymotryptic agents [13]. As a result, PIM-1 kinase has been turned into one of the attractive targets for the design of anticancer drugs recently [14]. Since then, many designed chemical compounds have found their way into the clinical trials $[15,16]$. One of the chemical compounds which is suggested as the inhibitor of PIM-1 is (R, Z)-5-((2-(3-aminopiperidin-1-yl)-[1,1'-biphenyl]-3-yl) methylene) thiazolidine-2,4-dione (AZD1208), which can be seen in Figure 1.

Figure 1. Structure of (R, Z)-5-((2-(3-aminopiperidin-1-yl)-[1,1'-biphenyl]-3-yl) methylene) thiazolidine2,4-dione (AZD1208).

But, the AZD1208 like other chemical drugs have been reported to have unwanted side effects such as Gastrointestinal problems, vascular disorders and Respiratory disorders [17]. Additionally, Gudong Li and et al. have designed and synthesised the novel chemical structure with a tricyclic scaffold (2-chloro-10-(5ethylheptyl)-10H-phenoxazine) to selectively inhibit PIM-1 kinase (Figure 2a) [18]. Moreover, they have modified the molecule in Figure 2(a) to achieve the most potent compounds as inhibitors of PIM-1 kinase including 2-(4-(2-(7-chloro-5H-benzo[b]pyrido[4,3-e] [1,4] oxazin-5-yl) ethyl) piperidin-1-yl) ethan-1-amine (Figure 2b) and 2-(4-(2-(7-bromo-5H-benzo[b]pyrido[4,3-e][1,4]oxazin-5-yl)ethyl) piperidin-1-yl)ethan-1-amine (Figure 2c) [19].

Figure 2. Tricyclic based structures as selective inhibitors of PIM-1 kinase including (a) 2-chloro10-(5-ethylheptyl)-10H-phenoxazine, (b) 2-(4-(2-(7-chloro-5H-benzo[b]pyrido[4,3-e] [1,4] oxazin-5-yl) ethyl) piperidin-1-yl) ethan-1-amine, and (c) 2-(4-(2-(7-bromo-5H-benzo[b]pyrido[4,3-e] [1,4] oxazin-5-yl) ethyl) piperidin-1-yl) ethan-1-amine.

The chemical structures designed to have inhibitory activity against PIM-1 kinase are expected to have side effects; moreover, in the design of new drugs against PIM-1 kinase, the limitation on dosage should be considered [17]. Also, treatment combinations for inhibiting PIM-1 kinase are used in many cancer types; such as, Prostate cancer (PCa), thus the safe and tolerable dose of PIM-1 kinase inhibitors are defined due to their toxicities [20]. Accordingly, our research group put their concentration on the computational design of novel drugs based on amino acids and carbohydrates to enable the cancer treatment with less worry about the dosage limits of medicines and their side effects. Up to now, many vaccines based on carbohydrates are synthesized, such as those to activate independent T-cell humoral responses, which lead to the formation of antibodies to mobilize immunoglobulin G [21]. Additionally, Carbohydrate-based anticancer vaccines have gained much attention over the last decades [22-24]. In addition to carbohydrates, therapeutic peptides cast scientists' attentions over themselves to be used as anticancer agents as these structures bearing some desirable features including the ability to penetrate cell membranes, high activity, specificity, affinity, and minimal drug-drug interaction. Also, therapeutic peptides are biologically diverse and are easy to be synthesized $[25,26]$. To maintain both carbohydrate and amino acids building blocks in the suggested drugs to inhibit PIM-1 kinase, we designed structures including amino acids alongside with the monosaccharides such as D-fructose. The synthesis of these structures can be achieved by Amadori and Heyns methods [27]. Although, clinical and experimental studies over the interactions of drugs and their receptors are proved to be effective; still some problems associated with these experimental researches including high costs, limited duration of effective treatment, unwanted immunogenicity associated with immunological resistance toward these drugs have not been solved yet $[28,29]$. Moreover, some details of biomolecular dynamics are not accessible through experiment [30]. We hope that due to the development of in silico techniques to study biomolecular systems, we can cut our path to drug discovery [31-36]. The current project was divided into five sections: 1) all of the structures based on amino acids and monosaccharides were optimized at B3LYP/6$311++\mathrm{G}(\mathrm{d}, \mathrm{p})$ level [37]; 2) single-point electronic energy for optimized geometries were calculated at 
B3LYP/6-311++G (d, p) level by using Spartan software [37] to calculate some physical properties of our suggested structures such as volumes, areas, and electrostatic potential maps of the structures. These features were compared with those of the currently reported drugs including the structures $\mathbf{b}$ andc in Figure 2 [19] to select our amino acid- and carbohydrate-based structures which are the most similar to the structures $\mathbf{b}$ and $\mathbf{c}$ in Figure 2; 3) the binding affinities of $\mathbf{b}$ and $\mathbf{c}$ in Figure 2 and our selected structures toward PIM-1 kinase were calculated by Auto Dock/Vina plugin with scripts from the Auto Dock Tools Package [38]. Finally, the extracted data from the previous steps were used as inputs for MD simulation (The GROMCS 5.2) [39]. Then MM-PB/SA method was used to achieve binding energies between ligands and receptor [40].

\section{Method and theoretical calculations}

\subsection{Molecular docking and Geometry optimizationsof the suggested structures as anticancer drugs}

Before initiating the molecular docking process, all the molecules were optimized to ensure that all structures have acceptable properties including electronic properties, bond lengths, angles, and proper dihedrals. To find the most stable conformers of our amino acid- and carbohydrate- based structures, the initial search of minima on the potential energy surface were carried out using the Merck Molecular force field (MMFF) by the aid of the Spartan software [37]. Then, the geometry optimizations and frequency calculations were performed on the most stable conformers using the $6-311++\mathrm{G}(\mathrm{d}, \mathrm{p})$ basis set [37]. The absence of imaginary frequencies confirmed that the optimized structures corresponded to the real minima. Moreover, the $6-311++\mathrm{G}(\mathrm{d}, \mathrm{p})$ basis set contain polarized and diffusive functions, which pursue our goals to studying the intramolecular hydrogen bonds.

The nomenclatures and codes of molecules studied herein are listed in Table 1. For example, DBC is the code of the structure shown in Figures $2 \mathrm{~b}$ and $3 \mathrm{a}$ with the name of 2-(4-(2-(7-chloro-5H-benzo[b]pyrido[4,3-e] $[1,4]$ oxazin-5-yl) ethyl) piperidin-1-yl) ethan-1-amine; and UNK0 is the code of our suggested structure with the name of ((2S,3R,4R,5S,6R)-2,4,5-trihydroxy-6-(hydroxymethyl) tetrahydro-2H-pyran-3-yl)-L-valine, The structure of DBC extracted from the PDB ID of 6L16 is shown in Figure 3a and the structures of our suggested drugs including UNK0, UNK1,UNK2,UNK3,UNK4,UNK5 and UNK6 optimized at B3LYP/6-311++G (d, p) level can be seen in Figures 3b-3h. To make a good comparison between DBC and suggested structures, we obtained the electrostatic potential maps, volume, and area of these structures (see Table 1). 


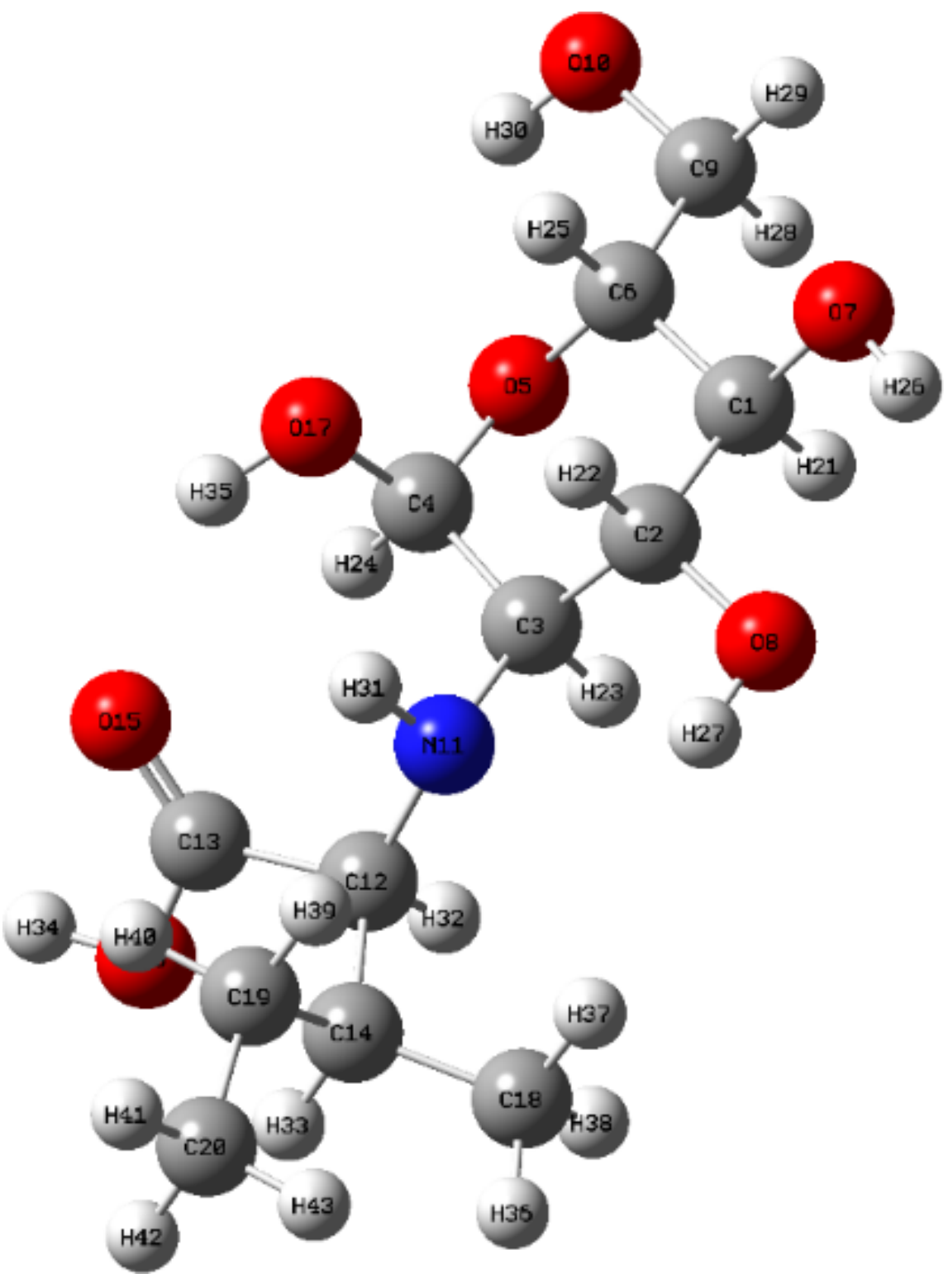



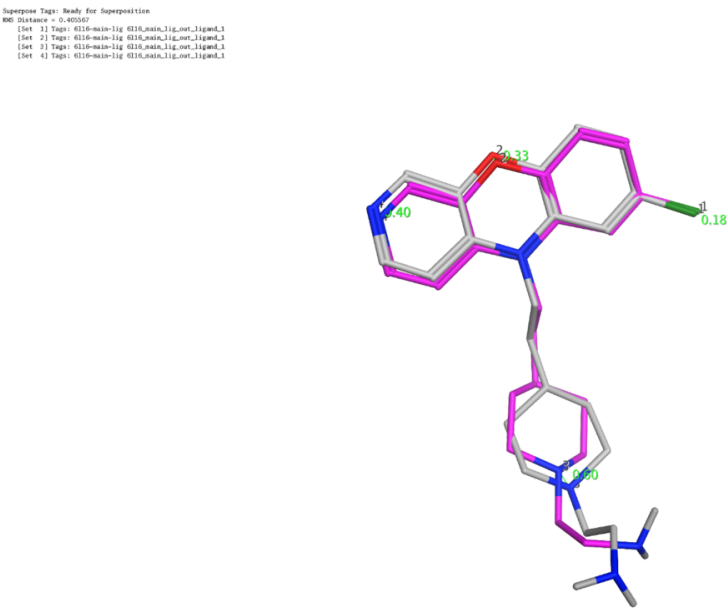

Before starting the docking procedure, the complex of protein and DBC was edited by VMD package [41]. Additionally, water molecules, other solvents, and other co-crystallised ligand were removed by use of Discovery studio 4.5. [42]. Before calculation of affinities by Auto Dock/Vina, the PDB files of the receptor and DBC should be transformed into the pdbqt file format by auto dock tools [43]. Pdbqt files mostly include the data such as atomic charges, atom-type definitions, and rotatable bonds (like single bonds in molecules' structures) [44]. The docking procedure (Auto Dock/Vina) started by using grid box with dimensions of $22 \times 28 \times 26 \AA^{3}$ with its center located at $\mathrm{x}=24.955, \mathrm{y}=119.79, \mathrm{z}=5.32$. The obtained results from the mentioned analyses can be seen in Table 1 . The term $E_{\text {scf }}$ in Table 1 refers to the Absolute energies $\left(\mathrm{E}_{\mathrm{SCF}}, \mathrm{au}\right)$ [37]. Comparison of entries 1 and 6 in Table 1 indicates DBC and UNK4 (UNK4 contains fructose and tryptophan) have volumes $=382.11,344.14 \AA^{3}$, respectively; and their areas $=400.61$, and $366.93 \AA^{2}$, respectively. Therefore, the ratios of area/volume $=1.048$, and 1.066 for DBC and UNK4, respectively. According to the calculations of EPS, it is expected that two structures have similarities in the electron acceptor and electron donor parts. These calculated features indicate the similarities between DBC and UNK4. In this study, to validate the auto dock procedure and to ensure that the dimensions of grid box are assigned appropriately, the output structure of docking for DBC and the DBC structure extracted from primary PDB were superposed, and the resulted RMSD was calculated to be $0.405567 \AA$, which is $<1.2 \AA$ and is acceptable $[45,46]$ (Figure 4).

Table 1. The affinity results of PIM-1 kinase obtained by Auto dock vina for DBC and the suggested amino acid- and carbohydrate-based structures (UNK0-UNK6); $\mathrm{E}_{\mathrm{scf}}$ values for UNK0-UNK6 calculated at B3LYP/6-311++ G (d, p) level; area, volume, and electrostatic potential maps for DBC and UNK0-UNK6. 


\begin{tabular}{|c|c|c|c|c|c|c|c|}
\hline Entry & $\begin{array}{l}\text { Name of } \\
\text { structures }\end{array}$ & $\begin{array}{l}\text { Structures' } \\
\text { codes }\end{array}$ & $\begin{array}{c}\mathbf{E}_{\mathrm{SCF}} \\
(\text { Hartree/Par }\end{array}$ & ttikedegptor & $\begin{array}{l}\text { AFFINTY } \\
(\mathrm{kcal} / \mathrm{mol})\end{array}$ & Area $\AA^{2}$ & $\begin{array}{l}\text { Volume } \\
\AA^{3}\end{array}$ \\
\hline 1 & $\begin{array}{l}2-(4-(2-(7- \\
\text { chloro-5H- } \\
\text { benzo[b]pyrido[4,3- } \\
\text { e] }[1,4] \\
\text { oxazin-5- } \\
\text { yl) ethyl }) \\
\text { piperidin- } \\
\text { 1-yl) } \\
\text { ethan-1- } \\
\text { amine }\end{array}$ & $\mathrm{DBC}$ & $\begin{array}{l}\text { Extracted } \\
\text { from x-ray } \\
\text { crystallog- } \\
\text { raphy } \\
\text { PDB ID: } \\
6 \text { L16 }\end{array}$ & $\begin{array}{l}\text { PIM-1 } \\
\text { kinase }\end{array}$ & -9.0 & 400.61 & 382.11 \\
\hline 2 & $\begin{array}{l}((2 \mathrm{~S}, 3 \mathrm{R}, 4 \mathrm{R}, 5 \mathrm{~S}, 6 \mathrm{R})- \\
2,4,5- \\
\text { trihydroxy- } \\
6- \\
\text { (hydroxymethyl) } \\
\text { tetrahydro- } \\
2 \mathrm{H}- \\
\text { pyran- } \\
3-y \mathrm{l})- \\
\mathrm{L}- \\
\text { valine }\end{array}$ & UNK0 & $\begin{array}{l}- \\
1013.11242\end{array}$ & $\begin{array}{l}\text { PIM-1 } \\
\text { kinase }\end{array}$ & -5.6 & 291.53 & 263.44 \\
\hline 3 & $\begin{array}{l}((2 \mathrm{~S}, 3 \mathrm{R}, 4 \mathrm{R}, 5 \mathrm{~S}, 6 \mathrm{R})- \\
2,4,5- \\
\text { trihydroxy- } \\
6 \text { - } \\
\text { (hydroxymethyl) } \\
\text { tetrahydro- } \\
2 \mathrm{H}- \\
\text { pyran- } \\
3 \text {-yl)- } \\
\text { L- } \\
\text { arginine }\end{array}$ & UNK1 & $\begin{array}{l}- \\
1217.290564\end{array}$ & $\begin{array}{l}\text { PIM-1 } \\
\text { kinase }\end{array}$ & -6.3 & 336.01 & 308.53 \\
\hline 4 & $\begin{array}{l}((2 \mathrm{~S}, 3 \mathrm{R}, 4 \mathrm{R}, 5 \mathrm{~S}, 6 \mathrm{R})- \\
2,4,5- \\
\text { trihydroxy- } \\
6- \\
\text { (hydroxymethyl) } \\
\text { tetrahydro- } \\
2 \mathrm{H}- \\
\text { pyran- } \\
3-\mathrm{yl} \text { - } \\
\text { L- } \\
\text { isoleucine }\end{array}$ & UNK2 & $\begin{array}{l}- \\
1052.407997\end{array}$ & $\begin{array}{l}\text { PIM-1 } \\
\text { kinase }\end{array}$ & -5.9 & 312.44 & 282.33 \\
\hline
\end{tabular}




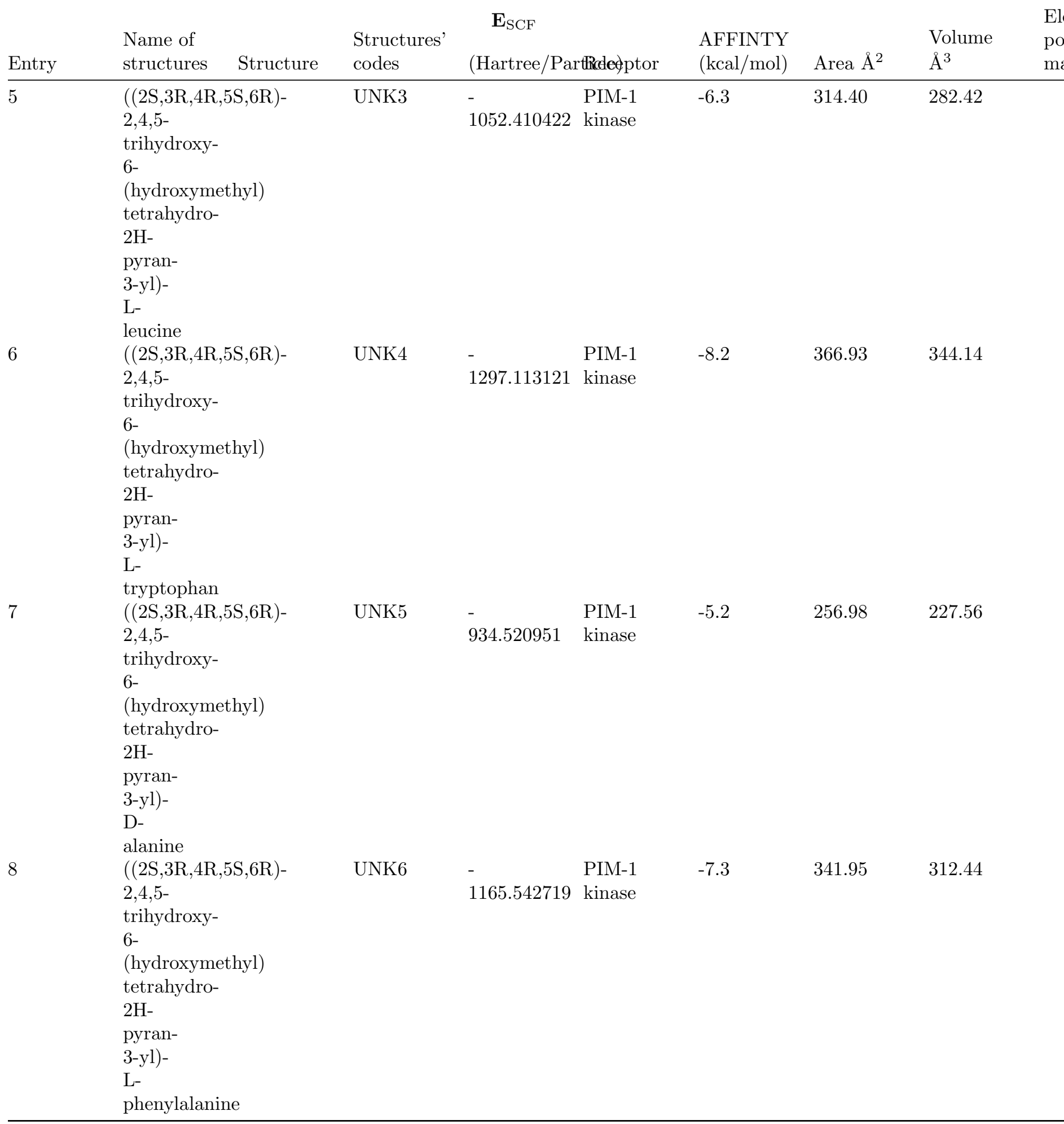


To add more emphasis on accuracy of our choice of UNK4 as a suitable replacement for DBC (see Table 1 for the codes), we also performed grid box searching process using Auto Dock 4.2.2 software [47] to find the best active site of PIM-1 kinase for UNK4 and compared it with that for DBC. The spacing between grid points was considered to be $0.375 \AA$, and the grid box size was set at126 $\times 126 \times 126 \AA^{3}$; UNK4 as a ligand was deemed to be flexible and the grid searching was carried out at local search genetic algorithm (LGA) $[48,49]$. The results obtained in this part of the project was in good agreement with the results of Auto dock/vina. The selected initial structure of UNK4 for the MD simulation was the most populated conformation in each cluster and the best pose with the most negative binding energy. Figures $5 \mathrm{a}$ and $5 \mathrm{~b}$ illustrate the best pose of UNK4 and DBC in the pocket site of the PIM-1 receptor. As seen in Figure 5a, UNK4 molecule has interaction with residues of PIM-1 kinase including leucine 44, phenylalanine 49, valine 52 , alanine 65 , lysine 67, Isoleucine 104, aspartic acid 186, glutamic acid 121, and arginine 122. Also, in Figure 5(b), which is obtained according to the primary pdb structure (PDB ID: 6L16), DBC interacts with the same residues of PIM-1 kinase as UNK4 does.

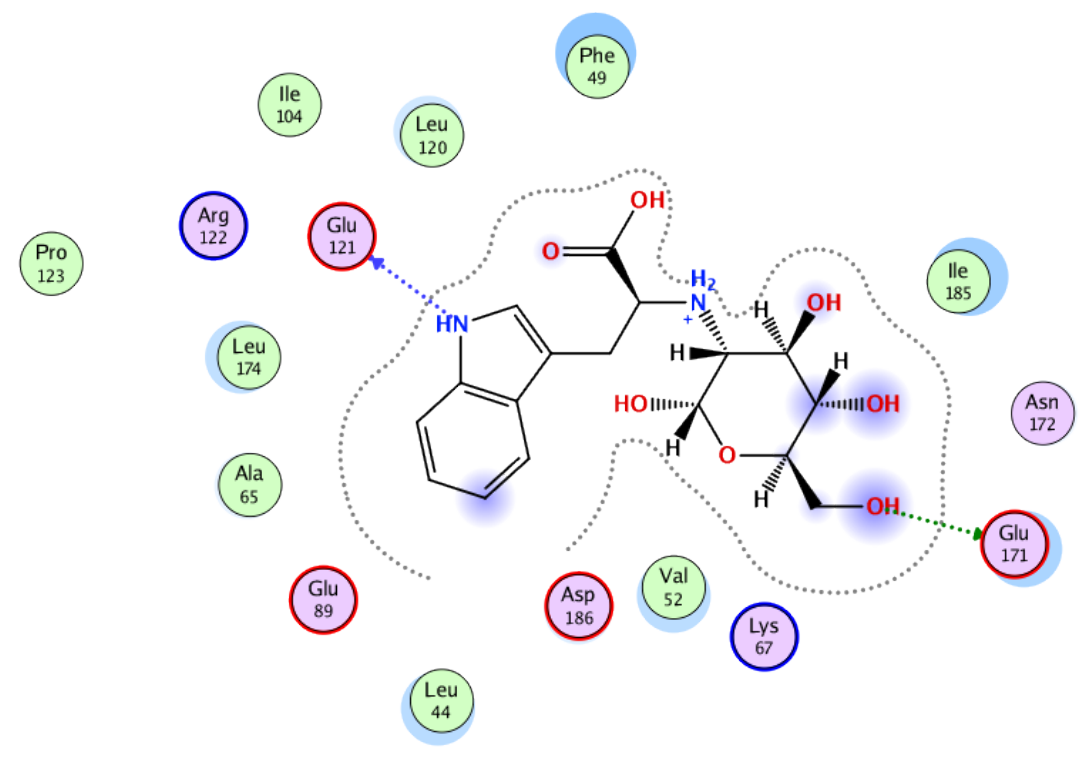

\subsection{Prediction of logarithm of the partition coefficient or $\log P$ of the suggested structures as anticancer drugs}

To predict the $\log \mathrm{P}$ value of the suggested structure UNK4 (with the best affinity), we used the structures listed in the entries 1-9 in Table $\mathrm{S} 1$ and their experimentally measured $\log \mathrm{P}$ values. We also used OSIRIS property explorer to calculate the $\log \mathrm{P}$ of these known compounds [50], which are shown by $\operatorname{cog} \mathrm{P}$ (c stands for calculated) in Table S1. Figure S1 represents the plot of the experimentally measured $\log \mathrm{P}$ values versus clogP for the compounds listed in the entries 1-9 in Table S1 with $y=1.019 x-0.117$ and $R^{2}=0.966$; where $y$ and $x$ indicate the experimental and calculated $\log \mathrm{P}$ values, respectively. Using this linear equation and calculated $\log \mathrm{P}$ of UNK4 $(\operatorname{cog} \mathrm{P}=-3.09)$, we then predicted the experimental value of $\log \mathrm{P}$ for $\mathrm{UNK} 4$ to be $\log \mathrm{P}=-3.26$.

\section{Molecular dynamic simulation}

MD simulation was carried out in two stages for the complex of ((2S,3R,4R,5S,6R)-2,4,5-trihydroxy-6(hydroxymethyl) tetrahydro-2H-pyran-3-yl)-L-tryptophan (UNK4) and PIM-1 kinase as well as the complex of (2-(4-(2-(7-chloro-5H-benzo[b]pyrido[4,3-e] [1,4] oxazin-5-yl) ethyl) piperidin-1-yl) ethan-1-amine) (DBC) and PIM-1 kinase as a receptor. The topology files for UNK4 and DBC were constructed based on the Gromos 43 a1 force field using the PRODRG 2.x online server. Two complexes were solvated by a layer 
of SPC water [51] in all directions. The simulation box was considered to be a triclinic box with faces at least $1.0 \mathrm{~nm}$ apart from the closest atom of our system. For the system including DBC and PIM-1 at least 9144 water molecules were considered as solvent molecules in the system; additionally, 15 sodium ions were added to the system for neutralization. The suggested system including UNK4 and PIM-1 kinase was located in a triclinic box with faces at least $1.0 \mathrm{~nm}$ apart from the closest atom of the whole system with 9127 water molecules as the solvent. Like the previous system (DBC and PIM-1 complex), 15 sodium ions were added to UNK4 and PIM-1 complex to neutralize the system. MD simulations for minimization of both mentioned systems were performed in 3 steps. The first step included the minimization of the whole systems using the steepest descent, and then in the second and third steps of minimization, the conjugate gradient methods and the steepest descent were used, respectively. emtol value was considered to be 100.0 $\mathrm{kJ} / \mathrm{mol}$. nm. in both systems. Afterwards, overall systems were equilibrated in the NVT ensemble for 500ps at $100 \mathrm{~K}$ in this NVT ensemble. Temperature coupling times were $0.1 \mathrm{ps}$ in both complexes. This step was followed by NPT ensemble equilibration by using MD integrator for 1000 ps at $300 \mathrm{~K}$ and 1 bar, respectively. Berendsen algorithm was selected for both barostat and thermostat coupling algorithms in this step [52]. In NPT ensemble for increasing the systems' temperature from 100 to $300 \mathrm{~K}$, the velocities were raised based on the Maxwell-Boltzmann distribution [53]. At last, the MD production steps for our two systems were initiated at $300 \mathrm{~K}$ with a time step of $1 \mathrm{fs}$ and both MD procedures were performed in $140 \mathrm{~ns}$. It is notable that Parrinello-Rahman barostat [54] alongside with Nose-Hoover thermostat algorithms in MD simulation stages were used for pressure and temperature coupling, respectively [56]. The periodic boundary condition was used for NPT simulation stage. For calculation of van der Waals interactions, 'Cut-off' method was employed. In MD simulations, the van der Waals ( $r v d w)$ interactions were accompanied by neighbor list cut-off (rlist), where rvdw $>=$ rlist. The frequency of updating the list of neighbors was considered as 10ps. Also, the Particle Mesh Ewald (PME) model was exploited to estimate the electrostatic interactions [55,56]. As consideration of constraints on hydrogen bonds in systems, the LINCS algorithm was employed in all of the MD simulations [57].

\section{Results and discussions}

\subsection{Comparison of inhibitory effectsof UNK4 and DBC on receptor}

The goal of the MD simulation in this study was to more precisely determine ligand (i.e., UNK4 or DBC) and PIM-1 kinase interactions in a state close to the natural conditions. In addition to the obtaining terms such as binding energy of ligands and PIM-1 kinase, molecular dynamic simulation enables us to predict trajectory stability and flexibility of the complexes of the ligands and receptor. As a result, based on information obtained herein, we hope to introduce the efficient inhibitors of PIM-1 kinase based on natural products without significant side effects as a suitable replacement for the primary drug DBC or other traditional drugs, which are used to inhibit PIM-1 kinase. One of the parameters studied to investigate the stability of the complexes was the root-mean-square deviation (RMSD) of PIM-1 kinase in $140 \mathrm{~ns}$ of simulations. Comparison of RMSD values for the backbone of PIM-1 kinase in the presence of DBC (Figure 7a) with those of PIM-1 in the presence of UNK4 (Figure 7b) during $140 \mathrm{~ns}$ of MD simulation indicates no significant difference. 


\section{RMSD}

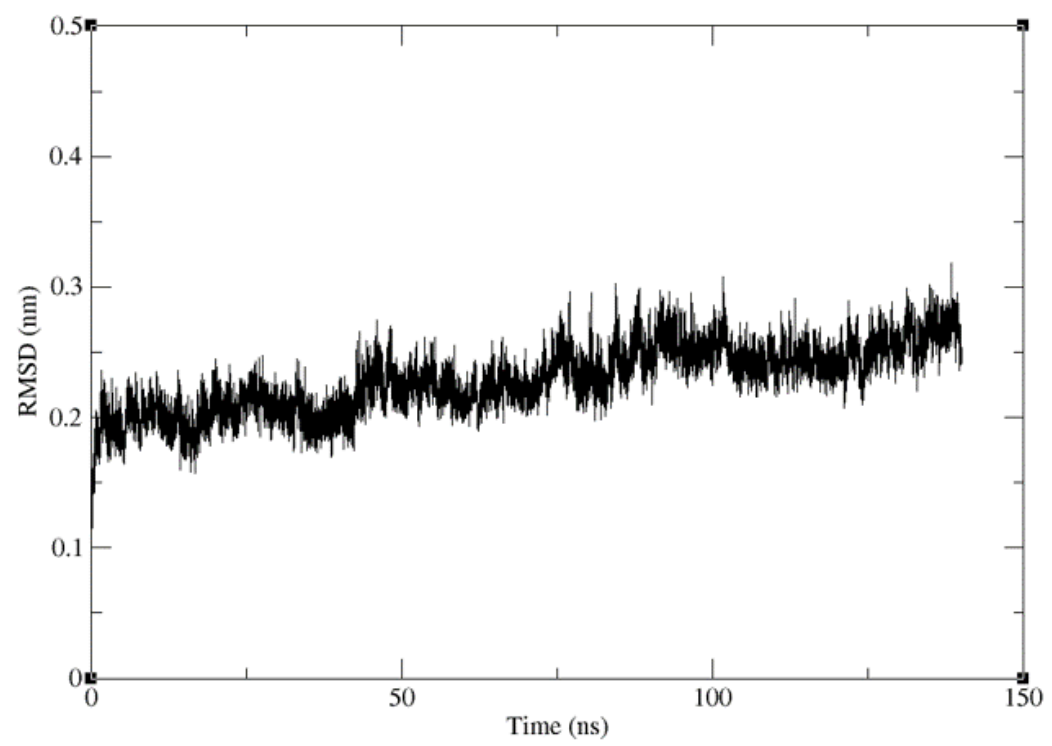

Additionally, to confirm the stability of the protein in both systems, root-mean-square fluctuation (RMSF) was obtained for protein in both systems. Most of the amino acids of the pocket site of protein are located between the residues 100150 (according to Figure 5), Figure 8a represents the RMSF values of PIM-1 kinase in complex with $\mathrm{DBC}$ for the residues 100 to 150 in the range of 0.18 to $0.25 \mathrm{~nm}$; and Figure $8 \mathrm{~b}$ indicates the RMSF values of PIM-1 kinase in the presence of UNK4 for the residues 100 to 150, which are mostly in the range 0.24-0.32 $\mathrm{nm}$. Based on the number of maxima in Figure 8, we can predict that the number of contacts between UNK4 and PIM-1 kinase is more than that between DBC and PIM-1 kinase during 140 ns of MD simulation, which can lead to the formation of a more stable complex between UNK4 and PIM-1 kinase. 


\section{RMS fluctuation}

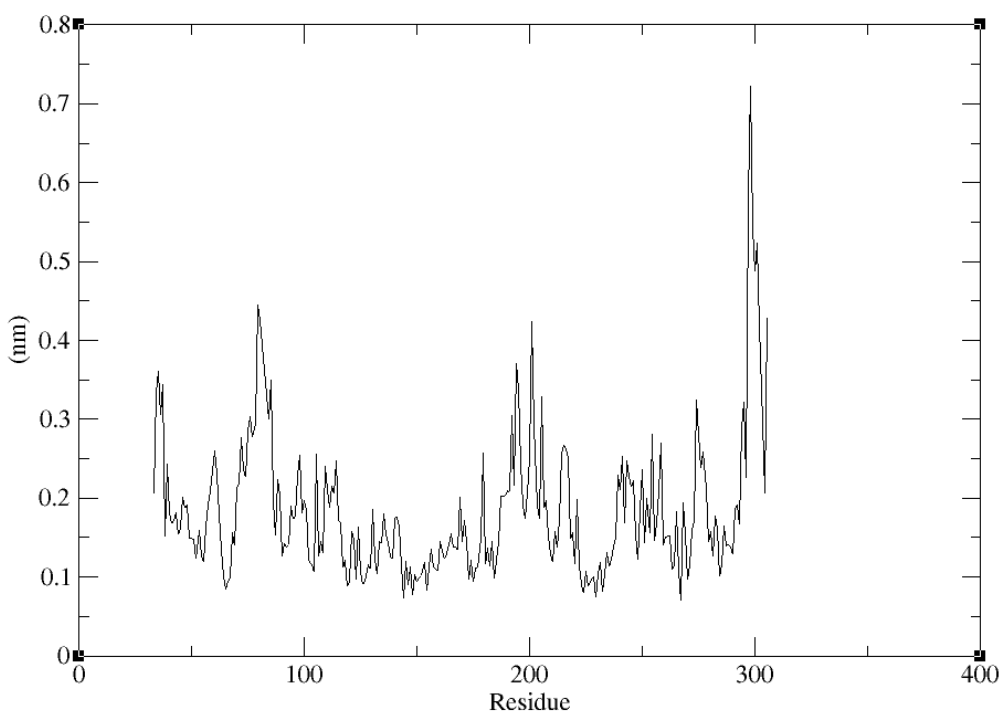

The compactness of systems during $140 \mathrm{~ns}$ of MD simulation was performed by measuring the radius of gyration of PIM-1 protein in complexes with DBC (Figure 9a) and UNK4 (Figure 9b). As seen in Figure 9, calculated $\mathrm{R}_{\mathrm{g}}$ for the complex of PIM-1 and Unk4 (Figure $9 \mathrm{~b}$ ) is lower than $\mathrm{R}_{\mathrm{g}}$ for the complex of PIM-1 and DBC, indicating that the complex of PIM-1-Unk4 is more compact than the complex of PIM-1-DBC. Moreover, the lower $\mathrm{R}_{\mathrm{g}}$ of the complex of PIM-1 and Unk4 indicates formation of a higher number of hydrogen bonds between PIM-1 protein and UNK4 compared to the number of hydrogen bonds between PIM-1 protein and DBC.

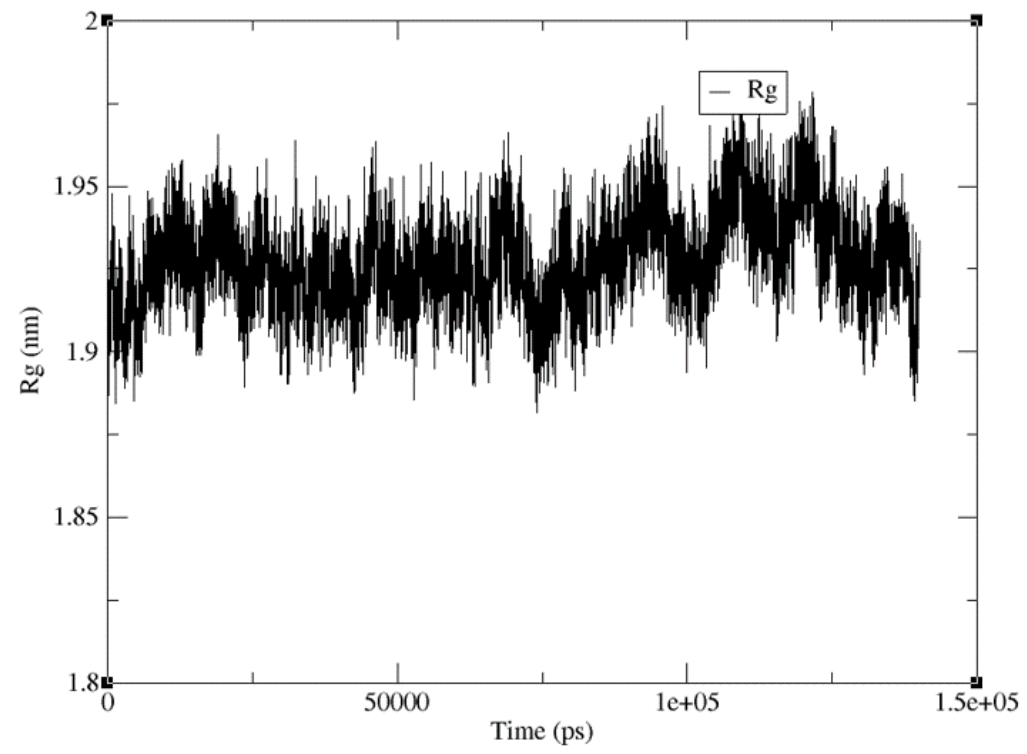


Figure 10 presents the number of hydrogen bonds between ligands (including DBC and UNK4) and receptor (PIM-1 kinase). As seen in Figure 8b, the number of hydrogen bonds between UNK4 and PIM-1 kinase during $140 \mathrm{~ns}$ of simulation is more than that between DBC and PIM-1 kinase. The results obtained here are in complete agreement with the results of the calculation of gyration radius (Figure 9).

\section{Hydrogen Bonds}

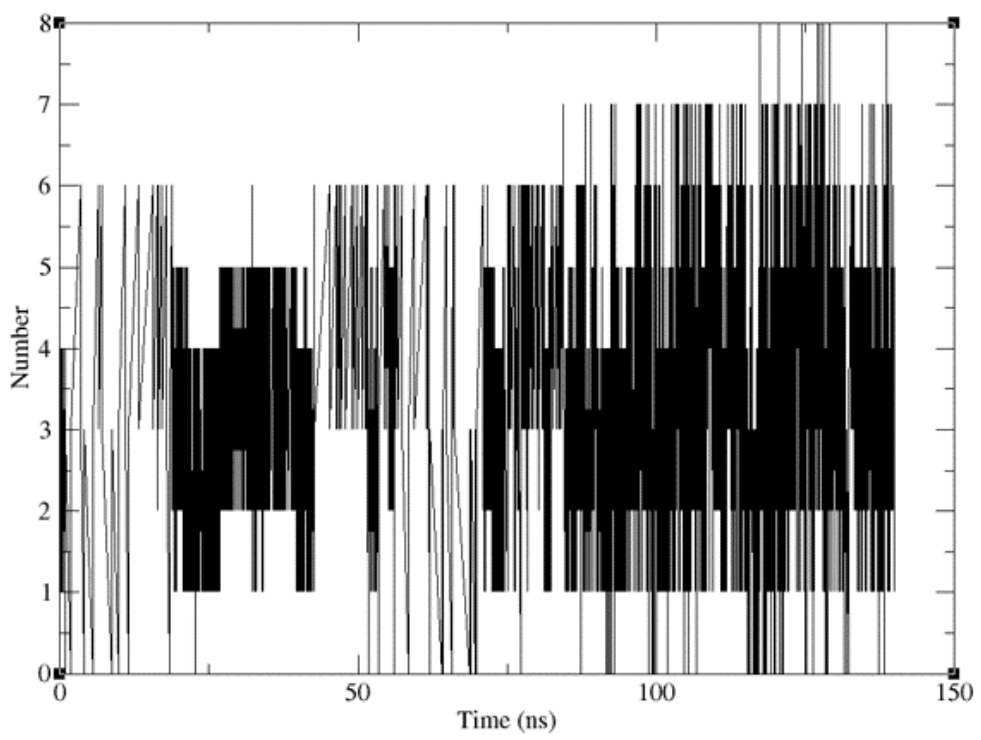

To better evaluate the stability of two complexes in this study, the fluctuation of minimum distances between ligands (DBC and UNK4) and PIM-1 kinase were plotted against time (Figure 11). From Figure 11(b), it can be inferred that the minimal distance between UNK4 and PIM-1 kinase is less than that between DBC and PIM-1 kinase (Figure 11a). This could be attributed to the formation of more hydrogen bonds between UNK4 and PIM-1 kinase (Figure 10b) in comparison with the number of hydrogen bonds between DBC and PIM-1 kinase (Figure 10a). As a result, the complex of UNK4 and PIM-1 kinase is expected to be more stable than complex of DBC and PIM-1 kinase. 


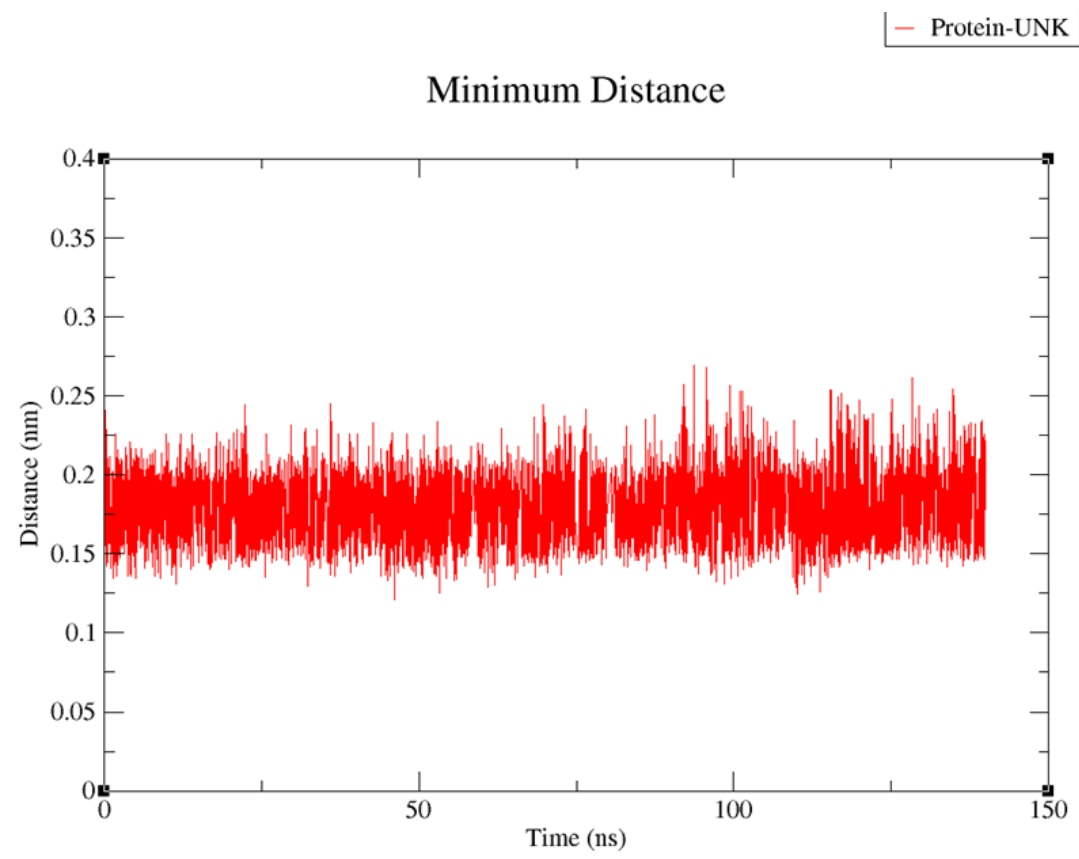

\section{2. $\mathrm{MM} / \mathrm{PBSA}$ calculation of UNK4 and DBC on receptor(PIM-1 kinase)}

The binding free energies of UNK4 and DBC on the receptor (PIM-1 kinase) were obtained by consideration of molecular Mechanics/Poisson-Boltzmann Surface Area (MM/PBSA) method by taking 1000 snapshots from the $140 \mathrm{~ns}$ period of MD trajectory. The energy terms calculated by g_mmpbsa package are listed in Table 2 [42]. MM/PBSA results illustrate that binding energy of UNK4 to PIM-1 kinase $(-204.21 \mathrm{~kJ} / \mathrm{mol})$ is more negative than that of DBC to PIM-1 kinase $-180.69 \mathrm{~kJ} / \mathrm{mol}$ ). According to our results, the binding energy values of both DBC and UNK4 are negative and their interactions with the receptor (PIM-1 kinase) are thermodynamically very favorable. As a result, UNK4, with the volume, area, and electron density similar to DBC, could be considered as an alternative anticancer drug for inhibition of PIM-1 kinase with much fewer side effects compared to traditional anticancer drugs because its building blocks are amino acid and cyclic carbohydrate.

Table 2. Various energy terms for the complexes of DBC and UNK4 with PIM-1 kinase obtained through MM/PBSA calculations.

\begin{tabular}{lll}
\hline MM/PBSA & PIM-1 kinase and DBC & PIM-1 kinase and UNK4 \\
\hline van der Waals energy $(\mathrm{kJ} / \mathrm{mol})$ & $-187.86 \pm 14.33$ & $-203.58 \pm 12.18$ \\
Electrostatic energy $(\mathrm{kJ} / \mathrm{mol})$ & $0.807 \pm 8.17$ & $-92.52 \pm 33.74$ \\
Polar solvation energy $(\mathrm{kJ} / \mathrm{mol})$ & $23.14 \pm 13.11$ & $110.24 \pm 17.06$ \\
Non-polar solvation energy $(\mathrm{kJ} / \mathrm{mol})$ & $-16.78 \pm 1.54$ & $-18.35 \pm 0.85$ \\
Binding energy $(\mathrm{kJ} / \mathrm{mol})$ & $-180.69 \pm 18.09$ & $-204.21 \pm 26.12$ \\
\hline
\end{tabular}

\section{Conclusion}

In summary, in this project, inhibition of PIM-1 kinase was studied by the aid of molecular dynamics simulations. The interaction of this protein with DBC, the newly designed and synthesized compound by Gudong Li and et al., was explored. We studied a novel inhibitor (UNK4) for PIM-1 kinase with the building 
block of amino acid and carbohydrate. Small molecules composed of D-fructose and amino acids such as valine, arginine, isoleucine, leucine, tryptophan, alanine, and phenylalanine were chosen as candidates to inhibit PIM-1 kinase. We used in silico studies to find the best candidates of these newly designed drugs. Our results indicated that our designed UNK4 drug with fructose and tryptophan building blocks (see Table 1 for the codes) could be a suitable replacement for DBC. This hypothesis was illustrated by steps including optimization of chemical structures at B3LYP/6-311++G (d, p) level and calculating single-point electronic energy for optimized geometries at B3LYP/6-311++G (d, p) level using Spartan software. Then, some physical properties of our suggested structures and DBC such as volumes, areas, and electrostatic potential maps were obtained and compared with each other to obtain the best designed candidates as alternatives for DBC to inhibit PIM-1 kinase.

Moreover, Auto Dock/Vina provided us with data such as binding affinities of UNK4 and DBC toward the receptor. To add more emphasis on accuracy of our choice of UNK4 as a suitable replacement for DBC, we also performed grid box searching process using Auto Dock 4.2.2 software. Also, newly designed molecules should have acceptable solvation in both lipophilic and hydrophilic media. OSIRIS property explorer enabled us to predict the $\log \mathrm{P}$ of UNK4. In addition, MD simulations (using GROMCS 5.2) provided the results to compare RMSD values for the backbone of PIM-1 kinase in the presence of DBC with those of PIM-1 in the presence of UNK4 during 140 ns of MD simulation.

It should be noted that other parameters such as the number of hydrogen bonds between ligands (including DBC and UNK4) and receptor (PIM-1 kinase) along with the fluctuation of minimum distances between ligands (DBC and UNK4) and PIM-1 kinase during MD simulation proved that UNK4 could be a more efficient inhibitor of PIM-1 than DBC. Energy analysis performed by MM/PBSA calculations indicated that UNK4 (binding energy $=-204.21 \mathrm{~kJ} / \mathrm{mol}$ ), containing the natural building blocks of amino acid and carbohydrate, interacts with PIM-1 kinase more effective than DBC (binding energy $=-180.69 \mathrm{~kJ} / \mathrm{mol}$ ). As a result of studies in this project, UNK4 could be considered an alternative anticancer drug for DBC with much fewer side effects due to building blocks including amino acid and cyclic carbohydrates.

AcknowledgementsThe support and resources from Sharif University of Technology are gratefully acknowledged.

\section{References}

1. Bachmann, M.; \& Möröy, T. The serine/threonine kinase Pim-1. INT. J. BIOCHEM. CELL. B. 2005 , $37,726-730$.

2. Saris, C. J.; Domen, J.; \& Berns, A. The pim-1 oncogene encodes two related protein-serine/threonine kinases by alternative initiation at AUG and CUG. EMBO J. 1991, 10 , 655-664.

3. Eichmann, A.;Yuan, L.; Breant, C.; Alitalo, K.; \& Koskinen, P. J. Developmental expression of pim kinases suggests functions also outside of the hematopoietic system. Oncogene.2000 , 19 , 1215-1224.

4. Hoover, D. S.; Wingett, D. G.; Zhang, J.; Reeves, R.; \& Magnuson, N. S. Pim-1 protein expression is regulated by its 5'-untranslated region and translation initiation factor elF-4E. Cell Growth and Differentiation-Publication American Association for Cancer Research.1997 , 8, 1371-1380.

5. Leverson, J. D.; Koskinen, P. J.; Orrico, F. C.; Rainio, E. M.; Jalkanen, K. J.; Dash, A. B.; .. \& Ness, S. A. Pim-1 kinase and p100 cooperate to enhance c-Myb activity. Mol. Cell.1998 , 2 , 417-425.

6. Cuypers, H. T.; Selten, G.; Quint, W.; Zijlstra, M.; Maandag, E. R.; Boelens, W.; .. \& \& Berns, A. Murine leukemia virus-induced T-cell lymphomagenesis: integration of proviruses in a distinct chromosomal region. Cell. 1984, 37, 141-150.

7. Amaravadi, R.; \& Thompson, C. B. The survival kinases Akt and Pim as potential pharmacological targets. J. Clin. Investig.2005, 115, 2618-2624.

8. Bachmann, M.; Kosan, C.; Xing, P. X.; Montenarh, M.; Hoffmann, I.; \& Moroy, T. The oncogenic serine/threonine kinase Pim-1 directly phosphorylates and activates the G2/M specific phosphatase Cdc25C.INT. J. BIOCHEM. CELL. B. 2006 , 38 , 430-443.

9. Chiang, W. F.; Yen, C. Y.; Lin, C. N.; Liaw, G. A.; Chiu, C. T.; Hsia, Y. J.; \& Liu, S. Y. Upregulation of a serine-threonine kinase proto-oncogene Pim-1 in oral squamous cell carcinoma. Int. J. 
Oral Maxillofac. Surg. $2006,35,740-745$.

10. Amson, R.; Sigaux, F.; Przedborski, S.; Flandrin, G.; Givol, D.; \& Telerman, A. The human protooncogene product p33pim is expressed during fetal hematopoiesis and in diverse leukemias. Proc. Natl. Acad. Sci. U.S.A. $1989,86,8857-8861$.

11. Dhanasekaran, S. M.; Barrette, T. R.; Ghosh, D.; Shah, R.; Varambally, S.; Kurachi, K.; ...; \& Chinnaiyan, A. M. Delineation of prognostic biomarkers in prostate cancer. Nature.2001 , 412, 822826.

12. Warfel, N. A.; Sainz, A. G.; Song, J. H.; \& Kraft, A. S. (2016). PIM kinase inhibitors kill hypoxic tumor cells by reducing Nrf2 signaling and increasing reactive oxygen species. Molecular cancer therapeutics , 15 (7), 1637-1647.

13. Chauhan, S. S., Toth, R. K., Jensen, C. C., Casillas, A. L., Kashatus, D. F., \& Warfel, N. A. PIM kinases alter mitochondrial dynamics and chemosensitivity in lung cancer. Oncogene.2020 , 39 , 25972611.

14. Le, B. T.; Kumarasiri, M.; Adams, J. R.; Yu, M.; Milne, R.; Sykes, M. J.; \& Wang, S. Targeting Pim kinases for cancer treatment: opportunities and challenges. Future Med. Chem.2015 , 7, 35-53.

15. Chen, L. S.; Redkar, S.; Taverna, P.; Cortes, J. E.; \& Gandhi, V. Mechanisms of cytotoxicity to Pim kinase inhibitor, SGI-1776, in acute myeloid leukemia. Blood, Am. J. Hematol.2011, 118 , 693-702.

16. Keeton, E. K.; McEachern, K.; Dillman, K. S.; Palakurthi, S.; Cao, Y.; Grondine, M. R.; ... ; \& Shen, M. AZD1208, a potent and selective pan-Pim kinase inhibitor, demonstrates efficacy in preclinical models of acute myeloid leukemia. Blood. 2014 , 123 , 905-913.

17. Cortes, J.; Tamura, K.; DeAngelo, D. J.; De Bono, J.; Lorente, D.; Minden, M.; .. \& \& Godin, R. Phase I studies of AZD1208, a proviral integration Moloney virus kinase inhibitor in solid and haematological cancers. Br. J. Cancer. $2018,118,1425-1433$.

18. Li, W.; Wan, X.; Zeng, F.; Xie, Y.; Wang, Y.; Zhang, W.; . . \& Huang, N. More than just a GPCR ligand: structure-based discovery of thioridazine derivatives as Pim-1 kinase inhibitors. Med.Chem.Comm. 2014, 5, 507-511.

19. Li, G.; Zhang, W.; Xie, Y.; Li, Y.; Cao, R.; Zheng, G.; . . \& Zhou, Y. Structure-Based Optimization of 10-DEBC Derivatives as Potent and Selective Pim-1 Kinase Inhibitors. J. Chem. Inf. Model. 2020 , $60,3287-3294$.

20. Luszczak, S.; Kumar, C.; Sathyadevan, V. K.; Simpson, B. S.; Gately, K. A.; Whitaker, H. C.; \& Heavey, S. PIM kinase inhibition: co-targeted therapeutic approaches in prostate cancer. Signal. Transduct. Target. Ther. 2020 , 5, 1-10.

21. Mond, J. J.; Lees, A.; \& Snapper, C. M. T cell-independent antigens type 2. Annu. Rev. Immunol. $1995,13,655-692$.

22. Geraci, C.; Consoli, G. M.; Galante, E.; Bousquet, E.; Pappalardo, M.; \& Spadaro, A. Calix [4] arene decorated with four Tn antigen glycomimetic units and P3CS immunoadjuvant: synthesis, characterization, and anticancer immunological evaluation. Bioconjug. Chem. 2008, 19 , 751-758.

23. Brooks, C. L.; Schietinger, A.; Borisova, S. N.; Kufer, P.; Okon, M.; Hirama, T.; .. \& \& Evans, S. V. Antibody recognition of a unique tumor-specific glycopeptide antigen. Proc. Natl. Acad. Sci. U.S.A. , 2010 , 107, 10056-10061.

24. Renaudet, O.; Dasgupta, G.; Bettahi, I.; Shi, A.; Nesburn, A. B.; Dumy, P.; \& BenMohamed, L. Linear and branched glyco-lipopeptide vaccines follow distinct cross-presentation pathways and generate different magnitudes of antitumor immunity. PLOS. ONE.2010, 5, 11216.

25. Marqus, S.; Pirogova, E.; \& Piva, T. J. Evaluation of the use of therapeutic peptides for cancer treatment. J. Biomed. Sci.2017, 24, 21.

26. J Boohaker, R.; W Lee, M.; Vishnubhotla, P.; LM Perez, J.; \& R Khaled, A. The use of therapeutic peptides to target and to kill cancer cells. Curr. Med. Chem. 2012, 19 , 3794-3804.

27. Chanda, D.; \& Harohally, N. V. Revisiting Amadori and Heyns synthesis: Critical percentage of acyclic form play the trick in addition to catalyst. Tetrahedron Lett. 2018,59 , 2983-2988.

28. Emens, L. A. Cancer vaccines: on the threshold of success. Expert. Opin. Emerg. Drugs. 2008 , 13 , 295-308. 
29. Hu, Z.; Ott, P. A.; \& Wu, C. J. Towards personalized, tumour-specific, therapeutic vaccines for cancer. Nat. Rev. Immunol. $2018,18,168$.

30. Kukol, A. Molecular modeling of proteins (Vol. 443). Totowa, NJ: Humana Press, 2008.

31. Selassie, C.; \& Verma, R. P. History of quantitative structure-activity relationships. Burger's medicinal chemistry and drug discovery. $2008,1-96$.

32. Aqvist, J.; Medina, C.; \& Samuelsson, J. E. A new method for predicting binding affinity in computeraided drug design.Protein Eng. Des. Sel. 1994, 7 , 385-391.

33. Bohm, H. J. The development of a simple empirical scoring function to estimate the binding constant for a protein-ligand complex of known three-dimensional structure. J. Comput. Aided Mol. Des.1994 , $8,243-256$.

34. Apostolakis, J.; \& Caflisch, A. Computational ligand design. Comb. Chem. High Throughput Screen.1999, 2, 91-104.

35. Talele, T. T.; Khedkar, S. A.; \& Rigby, A. C. Successful applications of computer aided drug discovery: moving drugs from concept to the clinic. Curr. Top. Med. Chem. 2010, 10, 127-141.

36. Vickers, N. J. Animal communication: when i'm calling you, will you answer too? Curr. Biol. 2017 , 27, 713-715.

37. Y. Shao, L. Fusti-Molnar, Y. Jung, J. Kussmann, C. Ochsenfeld, S. T. Brown, A. T. B. Gilbert, L. V. Slipchenko, S. V. Levchenko and D. P. O'Neill, Wavefunct. Inc., Irvine CA, 2011.

38. Seeliger, D., \& de Groot, B. L. Ligand docking and binding site analysis with PyMOL and Autodock/Vina. J. Comput. Aided Mol. Des. 2010, 24 , 417-422.

39. Pall, S.; Abraham, M. J.; Kutzner, C.; Hess, B.; \& Lindahl, E. Tackling exascale software challenges in molecular dynamics simulations with GROMACS. In International Conference on Exascale Applications and Software, Springer, Cham, 2014 , 3-27.

40. Kumari, R.; Kumar, R.; OpenSource Drug Discovery Consortium; \& Lynn, A. g_mmpbsa, A GROMACS tool for high-throughput MM-PBSA calculations. J. Chem. Inf. Model. 2014, 54, 1951-1962.

41. Humphrey, W.; Dalke, A.; \& Schulten, K. VMD: visual molecular dynamics. J. Mol. Graph. 1996 , $14,33-38$.

42. Systemes, D. BIOVIA, discovery studio modeling environment. Release 4.5. Dassault Systemes: San Diego, CA, 2015.

43. Huey, R.; \& Morris, G. M. Using AutoDock 4 with AutoDocktools: a tutorial. The Scripps Research Institute, USA, $2008,54-56$.

44. Trott, O.; Olson, A. J. AutoDock Vina: improving the speed and accuracy of docking with a new scoring function, efficient optimization, and multithreading. J. Comput. Chem.2010, 31, 455-461.

45. Kufareva, I.; \& Abagyan, R. Methods of protein structure comparison. In Homology Modeling , Humana Press, 2011, 231-254.

46. Sapundzhi, F. I.; \& Dzimbova, T. A. Computer modelling of the CB1 receptor by molecular operating environment. Bulg. Chem. Commun. 2018, 50, 15-19.

47. Morris, G. M.; Huey, R.; Lindstrom, W.; Sanner, M. F.; Belew, R. K.; Goodsell, D. S.; \& Olson, A. J. AutoDock4 and AutoDockTools4: Automated docking with selective receptor flexibility. J. Comput. Chem . 2009 , $30,2785-2791$.

48. Mansourian, M.; Mahnam, K.; Madadkar-Sobhani, A.; Fassihi, A.; \& Saghaie, L. Insights into the human A 1 adenosine receptor from molecular dynamics simulation: structural study in the presence of lipid membrane. Med. Chem. Res. 2015 , 24, 3645-3659.

49. Morris, G. M.; Goodsell, D. S.; Halliday, R. S.; Huey, R.; Hart, W. E.; Belew, R. K.; \& Olson, A. J. Automated docking using a Lamarckian genetic algorithm and an empirical binding free energy function.J. Comput. Chem. 1998, 19 , 1639-1662.

50. Sander, T. OSIRIS property explorer. Organic Chemistry Portal,2001 .

51. Berendsen, H. J.; Postma, J. P.; van Gunsteren, W. F.; \& Hermans, J. Interaction models for water in relation to protein hydration.In Intermolecular forces . Springer, Dordrecht, 1981, 331-342.

52. Davydov, A. S. Solitons in molecular systems . Dordrecht: Reidel, 1985, 113.

53. Roychoudhury, M.; Umesh Yadava, Bindesh Kumar Shukla. 
54. Berendsen, H. J. C.; \& Postma, J. P. M; Gunsteren, W.F.; van; DiNola, A.; Haak. J. Chem. Phys. 1984, 81, 3684-3690.

55. Yadav, R. K.; \& Yadava, U. Molecular dynamics simulation of DNA duplex, analog of PPT (polypurine tract), its conformation and hydration: a theoretical study. Med. Chem. Res. 2014,23, 280-286.

56. Darden, T.; York, D.; \& Pedersen, L. Particle mesh Ewald: An N[?] log (N) method for Ewald sums in large systems. J. Chem. Phys.1993, 98, 10089-10092.

57. Hess, B.; Bekker, H.; Berendsen, H. J.; \& Fraaije, J. G. LINCS: a linear constraint solver for molecular simulations. J. Comput. Chem. 1997, 18, 1463-1472.

\section{Hosted file}

graphical abstract.pdf available at https://authorea.com/users/394991/articles/508329design-of-amino-acid-and-carbohydrate-based-anticancer-drugs-to-inhibit-pim-kinases-anin-silico-study 